\title{
A Simulator for the Multi-model Control of Diesel Engines
}

\author{
Silviu CIRSTOIU, Dumitru POPESCU \\ Faculty of Automation and Computer Science, UPB, Bucharest, Romania, \\ silviu.cirstoiu@yahoo.com, popescu_upb@yahoo.com
}

\begin{abstract}
It was proposed and designed a modern configuration control type multicontroler-multimodel (MM) that pilots nonlinear combustion process of the diesel engine, needed to adjust the pressure in the intake manifold and air flow circulating through the compressor. The MM simulator developed by the authors allows the implementation of control systems represented by pairs $(\mathrm{Mi}, \mathrm{Ci})$ with the Mi candidate closest to the current operating point of the process and the paired controller $\mathrm{Ri}$, for controlling the key parameters of the combustion process. The proposed configuration is built with robust controllers and thus is able to ensure superior performance, tolerance to nonlinearities and parametric/ structural perturbations in the system.
\end{abstract}

Keywords: Diesel engine, dynamic models, optimal control, robust control, MM simulator.

\section{Introduction}

Multi-model control strategy for nonlinear systems configuration is a relatively new approach. In recent studies, one can find an ever increasing interest for management control of nonlinear processes or multimode operations, as diesel engines can only be roughly approximated by a single global model. The first works that have proposed solutions and methods suitable for this type of processes, relying mainly on the construction of adaptiverobust systems using classical algorithms are those of Balakrishnan and Narenda in the 90s[1]. Later detailed studies and positive results were presented by Athans (2006) who used the concept of multimodel control and demonstrated the effectiveness of this approach, with the risk of additional computation and implementation effort. The principle of building an MM configuration is the same, and is based on known identification procedures of Mi models and design of control algorithms $\mathrm{Ci}$, the differences are mainly due to the selection mode and command switching.

Studies by Petridis, Kehagias and Toscano use the multicontroler-multimodel (MM) configuration for systems with nonlinear static characteristics and Landau and Karimi use the so-called Cloe (Closed Loop Output Error) procedure, adjusting the parameters in an MM control structure [6]. Later studies have appeared on the use of neural networks and fuzzy logic systems, involved in the development of MM structures.

Research by the authors has been focused on proposing and testing control configurations MM for nonlinear processes implemented on certain applications designed to control the combustion process parameters in the diesel engines.

Following the preliminary results obtained in the paper, the proposed solution consists of a control structure (MM) in a robust version, to be tolerant to nonlinearities and disturbance regime of the diesel engine combustion.

The management configuration used here contains control loops (feedback) with pairs of models, robust controllers designed for the prespecified operating points of the nonlinear characteristic of the process, caused mainly by the variation engine load and actuators behavior.

We fix three possible operating points and for the designed $(\mathrm{Mi}, \mathrm{Ci}), \mathrm{i}=1,2,3$, systems, presumably linearized model $\mathrm{Mi}$ is disrupted in parameters and/or structure, there is a robustness analysis of systems in closed loop and adjust the nominal command but for all operating points preset. An adaptive strategy MM structure occurs when the engine is in operation between two operating points.

The multimodel control structure designed is shown in the Figure 1:

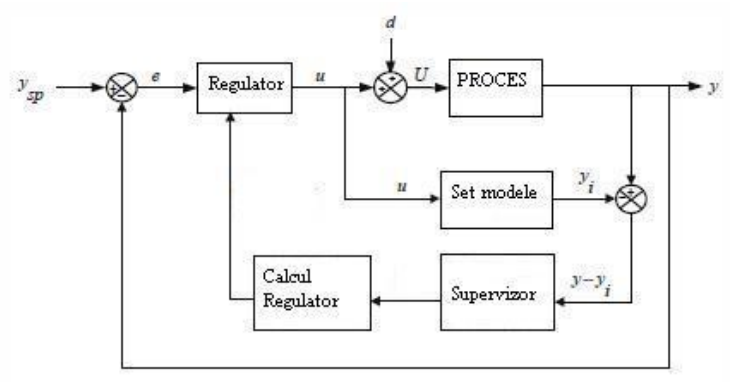

Figure 1. Multi model control structure 
where,

- $y s p$ - reference measure

- $\quad e$-the error between actual and desired output

- $u$-command

- $d$-disturbance

- $\quad U$-disturbed command

- $\quad y$ - the controlled output of the process

- $y i-\mathrm{i}$ model output

- $y-y i-$ difference between process output and i model output.

The supervising module must choose the right model and control algorithm for the engine functioning point. Thus we define the model error, calculated at each sampling moment and which represents the difference between output yi and output $y$ for the same $u$ value of the applied command.

$$
\hat{s}(k)=y(k)-y_{i}(k)
$$

The used criteria for selection of the closest model of the current process operating point is a square criteria built with the help of the model error, as in:

$$
J_{i}(k)=\alpha \alpha_{\xi}{ }^{2}(k)+\not \partial \sum_{j=1}^{k} e^{-\lambda[k-j]} e_{i}{ }^{2}(j)
$$

where $\alpha>0$ and $\beta>0$ are the criteria ponderation factors, and $\gamma>0$ is the forgiveness factor which ensures its action window limitation over the model error $\varepsilon_{i}(\mathrm{k})$.

The $\alpha, \beta$ and $\gamma$ parameters choice depends on the systems' characteristics, being:

- $\alpha=1$ and $\beta=0$, for fast response systems:

- better performances in detecting the process parameters modification,

- distrubances sensitivity,

- $\alpha=0$ and $\beta=0$, for slow response systems,

- weak performances in detecting the process parameters modification,

- good performances in detecting the process parameters modification.

The MM structure is configured for the proper version for controlling faster systems encountered in the case of controlling the working parameters of the diesel engine.

For each identified Mi model was computed a $\mathrm{Ci}$ regulator (controller) that meets the objectives and performance requirements; so we provide the set of pairs (Mi, Ci), corresponding to the possible nominal operating points $\mathrm{Pi}$. For $\mathrm{Ci}$ control algorithms, robust correction was applied.

\section{Building the MM Configuration}

To design effective control configuration MM one should consider the following issues: evidence of non-linear features in the configuration of the control system modelling study based on peculiarities processes in various points of operation, choosing the most suitable model for the dynamics of the process and algorithms, properly control the commutation MM system stability control[8]. A standard architecture for multi-model configuration is shown in Figure 2:

MM framing components in Figure 2 are:

- PROCESS-system to be controlled;

- BCC-block for calculating the order;

- SUPERVISOR System's state -component which gives information about the best matching of operating at a time and pair model-algorithm;

- M1, M2, ..., Mn-models for different operating points $r$;

- $\mathrm{C} 1, \mathrm{C} 2, \ldots, \mathrm{Cn}$-control algorithms

- SWITCH -switch control law;

- BSM-block selection most suitable model for the current state of the system based on predetermined criteria;

- y-process output;

- $\mathrm{y} 1, \mathrm{y} 2, \ldots, \mathrm{yN}$-output models;

- $\mathrm{u}$ and $\mathrm{u} 1, \mathrm{u} 2, \mathrm{UN}$-controls generated by BCC calculation block;

- r-reference system;

- p-physical disturbance process.

The overall structure can be sized according to the particularities and study system nonlinearities and switching according to procedures chosen by reconfiguration.

After specifying the operating points and the design of pairs (Mi, Ci), function switching control algorithms remain essential. The issue is the following: in what way and to what extent we can move from one control algorithm 


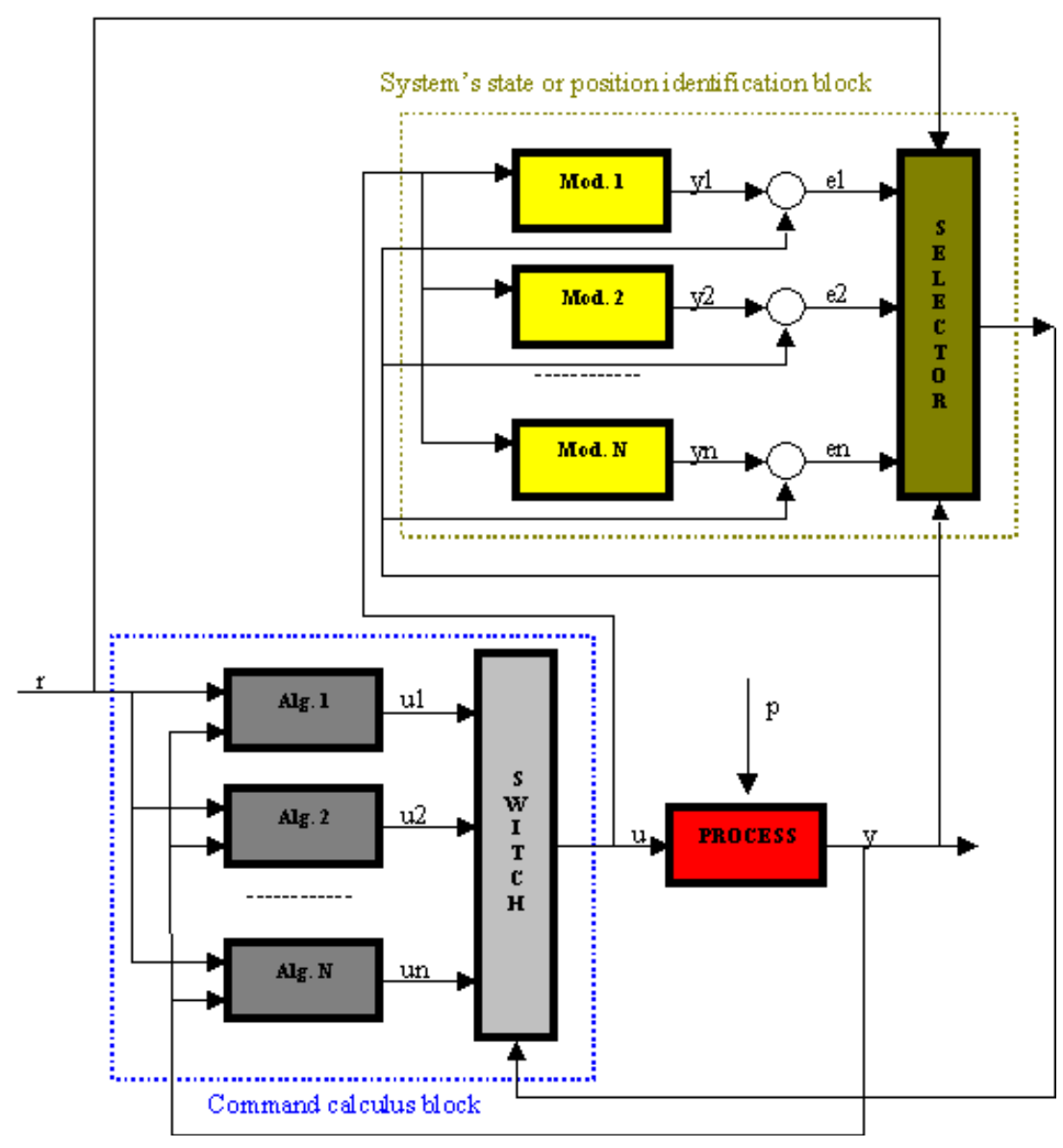

Figure 2. The classic Multi Model (MM) structure

obtained from another so that the stability offered by the initial performance that works in real time may be preserved. The novelty in this paper is how to calculate the pair (Mi, Ci) and propose a suitable solution for the switching operation of diesel engines using polynomial algorithms control type RST.

Therefore, by selecting the most suitable model for a particular mode of operation of the process and mapping pair adjustment algorithm, requires the algorithm switching between selected active algorithms at a given time.

The Engine disposes of a variable geometry turbine (VGT- Variable-Geometry Turbocharger), which allows efficient adaptation to the needs of the operating point of the engine equipped with exhaust gas recirculation valve, EGR (Exhaust Gas Recirculation) directing the gas flow outlet (residual) in the inlet in order to maintain a constant temperature in the combustion process. Although a technologically innovative solution, unfortunately the variable turbine geometry introduces nonlinear behavior. Therefore, the adopted
MM control design structure is justified for the control of the nonlinear regime. Thus we design the MM control solution for the key parameter: Wci airflow.

\section{MM System Configuration}

\subsection{The MM system for controlling the Wci airflow}

For the MM configuration design, we use the I/O model, given by the discrete transfer function:

$$
\begin{aligned}
& H d_{V G T-W_{c i}}(z)= \\
& \frac{0.03711 * z^{3}-0.0785 * z^{2}+0.09375 * Z+0.02185}{z^{3}-2.271 * z^{2}+1.617 * z-0.3458}
\end{aligned}
$$

The choice of the operating points was made taking into account the actuator nonlinearity (VGT turbine valve $x v$ ) that can operate through the opening in the range $0-40 \%$, 40$60 \%$ and $60-100 \%$. It is considered as a piecewise linearization around the operating points P1, P2, P3 corresponding to selected intervals and for each interval of domain partitioning drive $x v$ associate each segment tangent to the selected linear model. 
Under the reasoning of the previous section, we determine for each operating point, the corresponding pairs $(\mathrm{Mi}, \mathrm{Ci})$.

Using the CMMPR identification method and a sampling period $\mathrm{Te}=0.1 \mathrm{sec}$, on the software platform Adaptech / WinPE discrete linearized models are obtained, the entry $x v$ ordered at WCI adjustable output size:

M1, given by:

$H d_{E G R-p_{i}}(z)$

$=\frac{280.8 * z^{3}-102.9 * z^{2}-273.6 * z-99.22}{z^{3}-2.271 * z^{2}+1.617 * z-0.3458}$

M2, given by:

$H d_{E G R-p_{i}}(z)$

$=\frac{210 * z^{3}-56.8 * z^{2}-198.45 * z-77.28}{z^{3}-2.271 * z^{2}+1.617 * z-0.3458}$

$\mathrm{M} 3$, given by:

$H d_{E G R-p_{i}}(z)$

$=\frac{178.5 * z^{3}-12.7 * z^{2}-102.33 * z-24.44}{z^{3}-2.271 * z^{2}+1.617 * z-0.3458}$

Next it calls for the design platform Adaptech / Win Reg and pole assignment method to calculate the RST controllers.

The first operating point, corresponding to 0$40 \%$ range, we have the following results:

Controller computed with override $\mathrm{x} \_\mathrm{i}=0.95$ and natural pulsation $\mathrm{w}_{-} 0=10.5 \mathrm{rad} / \mathrm{sec}$ for tracking targets, respectively, $\mathrm{x} \_\mathrm{i}=0.95$,

w_0 $=12.5 \mathrm{rad} / \mathrm{sec}$ for setting targets, has the following components:

$$
\begin{aligned}
& R\left(z^{-1}\right)=-1.38-2.98 z^{-1}-0.11 z^{-2} \\
& S\left(z^{-1}\right)=1-0.96 z^{-1}-0.04 z^{-2} \\
& T\left(z^{-1}\right)=-8.45+4.77 z^{-1}-0.79 z^{-2}
\end{aligned}
$$

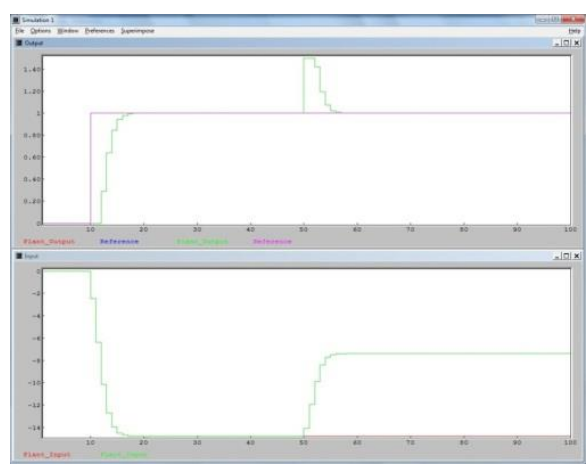

Figure 3. Nominal system response for the first operating point $\mathrm{P} 1$

New edge module is 0.72 , which means that by introducing a pair of zeros in the polynomial S, robustness of the system was improved.

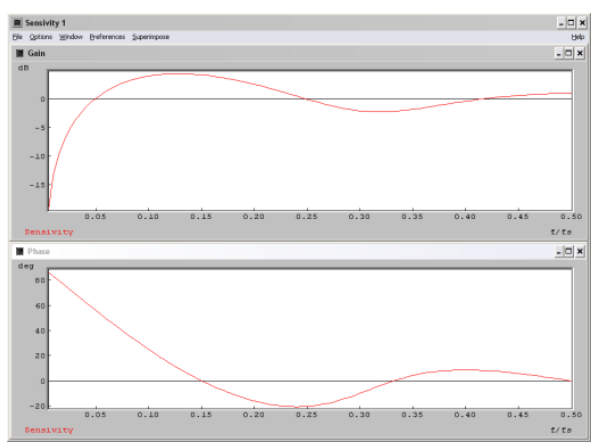

Figure 4. The sensitivity function andnthe phase characteristic of the robust system

Thus, for the second operating point corresponding range $40-60 \%$, we have the following results:

The controller calculated similarly with $\mathrm{x}_{-} \mathrm{i}=0.95 \mathrm{and} \mathrm{w} \_0=10.5 \mathrm{rad} / \mathrm{sec}$ for tracking goals and $\mathrm{x} \_\mathrm{i}=0.95$, w_0 $=12.5 \mathrm{rad} / \mathrm{sec}$ for setting goals, has the following components:

$R\left(z^{-1}\right)=-1.37-2.97 z^{-1}-1.09 z^{-2}$

$S\left(z^{-1}\right)=1-0.96 z^{-1}-0.037 z^{-1}$

$T\left(z^{-1}\right)=-8.42+4.75 z^{-1}-0.78 z^{-2}$

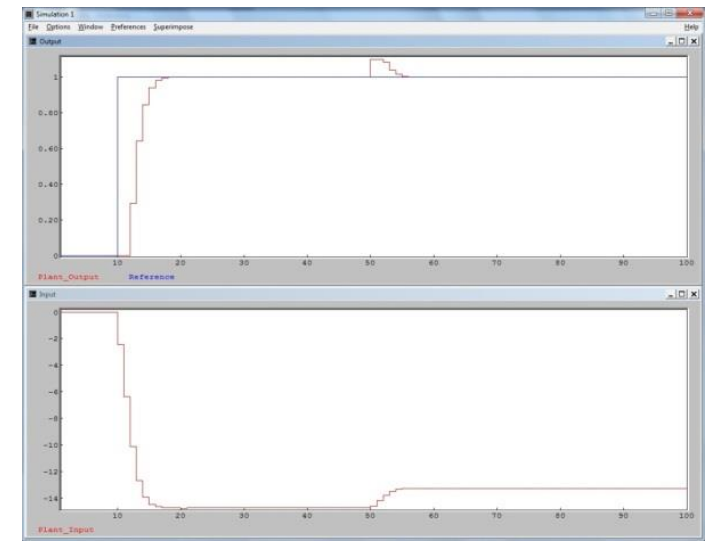

Figure 5. System response for the second functioning point $\mathrm{P} 2$

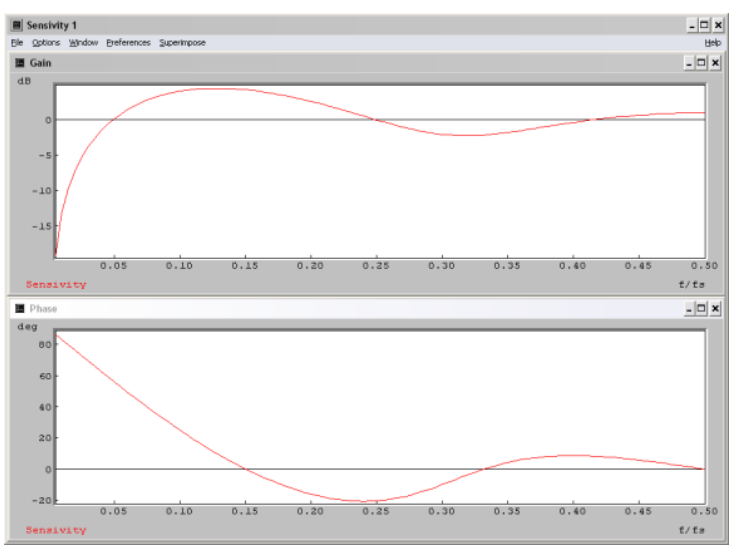

Figure 6. The sensitivity function andnthe phase characteristic of the robust system 
New edge module is 0.73 , which means that by introducing a pair of zeros in the polynomial $\mathrm{S}$, robustness of the system was improved.

For the third operating point corresponding range $60-100 \%$, we have the following results:

Controller computed with $\mathrm{x} \_\mathrm{i}=0.95$ and $\mathrm{w}_{-} 0=10.5 \mathrm{rad} / \mathrm{sec}$ for tracking goals and w_0 $=12.5 \mathrm{rad} / \mathrm{sec}$ with $\mathrm{x} \_\mathrm{i}=0.95$ for target setting, has the following components:

$R\left(z^{-1}\right)=-2.17-2.02 z^{-1}-0.33 z^{-2}$
$S\left(z^{-1}\right)=1-0.82 z^{-1}-0.17 z^{-2}$
$T\left(z^{-1}\right)=-8.56+4.83 z^{-1}-0.80 z^{-2}$

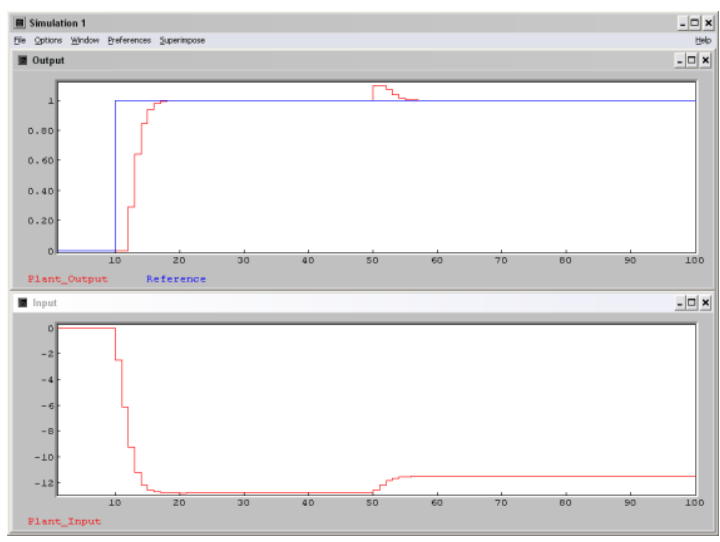

Figure 7. System response for the third functioning point $\mathrm{P} 3$

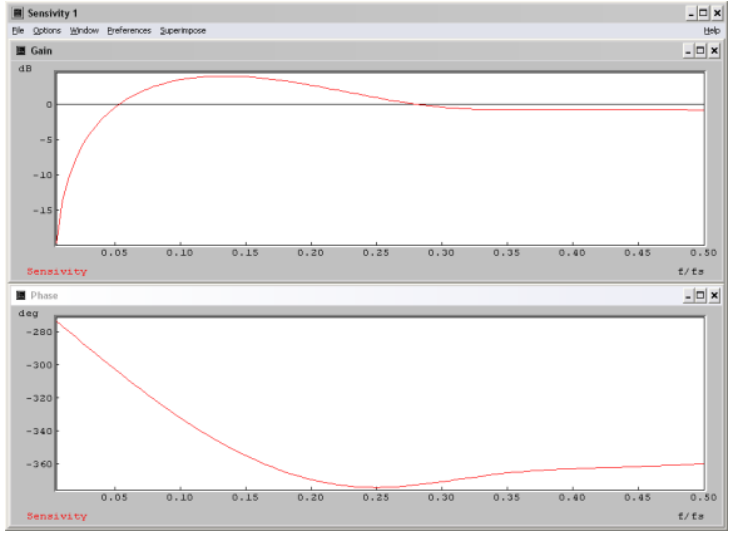

Figure 8. The sensitivity function andnthe phase characteristic of the robust system

The new edge module is 0.71 , meaning that the robustness of the system has been improved.

\section{The MM Application and Simulation Results}

After we have selected the operating points of the nonlinear characteristic associated to the motor functioning, there were determined the dynamic models (Mi-type I/O) and control algorithms (Ci type RST). The simulation was performed in closed loop systems to verify performance and ultimately the MM control structure was configured. The model selector was designed to specify the correct position in the real system operating conditions for the change point. It is based on the calculation error model and performance criteria.

$$
\begin{aligned}
& \varepsilon_{i}(k)=y(k)-y_{i}(k) \\
& J_{i}(k)=\alpha \varepsilon_{i}^{2}(k)+\beta \sum_{j=1}^{k} e^{-\lambda(k-j)} e_{i}^{2}(j)
\end{aligned}
$$

Regarding the switch control algorithm, switching is performed based on information received from the pattern selection, which builds a switching signal and calls for the new controller to be activated.

The three controllers are brought to active state, i.e. each controller receives the information as $\mathrm{y}(\mathrm{k})$ and reference $(\mathrm{k})$, and calculate their order without leaving $\mathrm{u}(\mathrm{k})$ to be applied.

For switching, the reference set will change given the output active control algorithm, and the resulting expression:

$$
y^{*}(k)=\frac{1}{t_{0}}\left[\sum_{i=0}^{n_{S}} s_{i} u(k-i)+\sum_{i=0}^{n_{R}} r_{i} y(k-i)-\sum_{i=1}^{n_{T}} t_{i} y^{*}(k-i)\right]
$$

with respect for the relation:

$y^{*}(k)=\frac{B_{m}\left(q^{-1}\right)}{A_{m}\left(q^{-1}\right)} r(k)$

The switching system will function according to the scheme below:

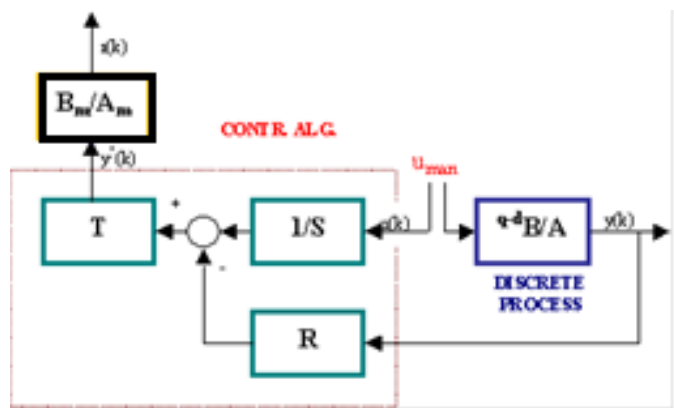

Figure 9. The set point value computation of the active algorithm using Uman command

Set point value will be identical to the active control algorithm when switching.

By using the solution of [9] we can eliminate the possibility of instability due to the probable gaps in the algorithm memory command.

It is therefore important to use the system memory command to always access the history in terms of switching between algorithms. 
To evaluate the simulation results, we use the software application CVI- National Instruments, which allows viewing configuration MM dynamics including control algorithms behavior in switching mode.

Simulation module is user friendly and realtime simulations are performed after all input data has been placed on the platform as shown in Figure 10, Figure 11, and Figure 12.

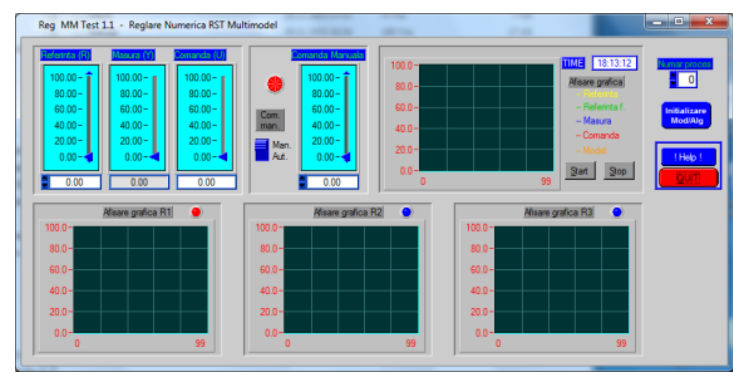

Figure 10. Software app for real time MM command

The application allows quick access to a range of information and commands, such as graphics display the time evolution of the process and control algorithms R1,R2,R3, highlighting the active algorithm, automatic or manual control of the system, the choice of study process, the possibility of initializing pairs obtained controlled models, the possibility of selection of references, etc.

The graphical display allows simultaneous viewing reference measure, control and response model, each corresponding color code.

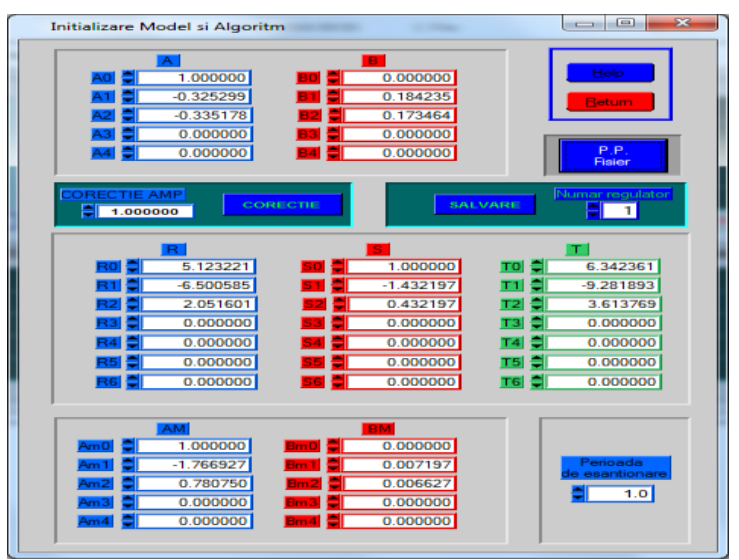

Figure 11. The initialization module for modelcontroller pairs

In the module initialization model, the control algorithm allows the introduction into the application of polynomial coefficients A and B models, and polynomials $\mathrm{R}, \mathrm{S}$ and $\mathrm{T}$ for controllers. After defining corrections amplification and sampling period, it generates actual simulation.
For example, we considered the case of the multimodel system for controlling the air flow Wci, in which case we consider three distinct operating pointsP1, P2 andP3, as reasoning from the beginning of the chapter; following the same rationale, we identified three models: M1 (0-40\%), M2 (40-60\%) and M3(60-100\%). For these models we have developed robust control algorithms, namely $\mathrm{C} 1, \mathrm{C} 2$, and $\mathrm{C} 3$.

Applying the superposition principle for pairs of models-algorithms to determine their control areas we get:M1(0-55\%), M2 (45-90\%) andM3(80-100\%).

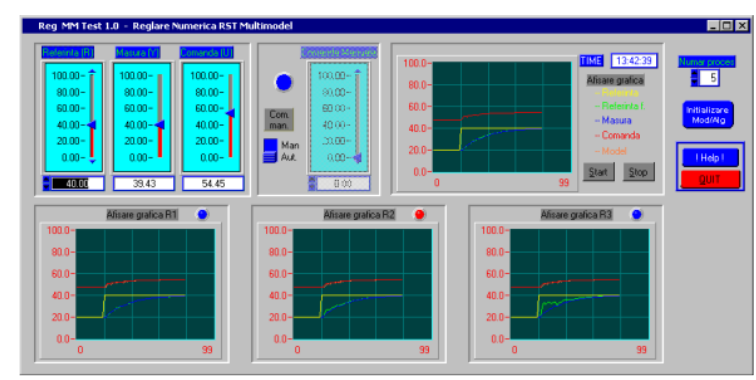

Figure 12. The MM system's response

Two switching tests were performed: the first in the range $40 \%-60 \%$, and the second in the range $80 \%-100 \%$, the results can be seen below.
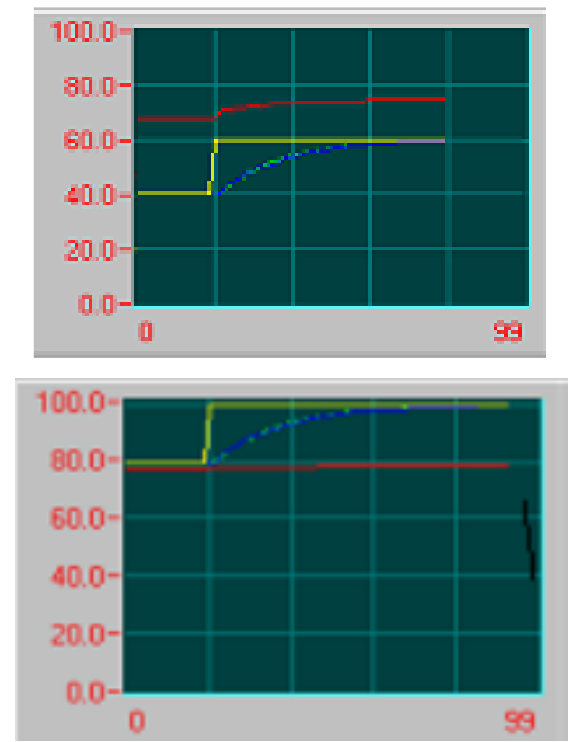

Figure 13. Switching tests

\section{Acknowledgment}

In this paper, an approach is considered for modeling and control of a turbocharged Diesel engine with EGR valve.

It was proposed and designed a configuration that steers multimodel MM-multicontroller nonlinear combustion process of diesel 
engine, available for adjusting the intake manifold pressure and air flow circulating through the compressor.

The MM software structure enables: implement systems represented by pairs $(\mathrm{Mi}, \mathrm{Ci})$, selecting the close of the current operation of the process and the controller pair, suitable for controlling air flow pressure pi and WCI order without affecting the stability of switching systems.

\section{REFERENCES}

1. BALAKRISHNAN., J., Control System Design Using Multiple Models, Switching and Tuning, PhD Thesis, Yale University, SUA, 1996.

2. DUMITRACHE, I., Ingineria Reglarii Automate, Politehnica Press, Bucharest, 2005, ISBN 973-8449-72-3.

3. LANGLOIS, N., Contribution à la modélisation et à la commande avancée des moteurs Diesel, 2008, Universite de Rouen.

4. LUPU, C., A. UDREA, D. POPESCU, C. FLUTUR, On the Stability of Switching in Multiple Model Control Systems, Politehnica University Bucharest. 2009a.

5. LUPU, C., D. POPESCU, B. CIUBOTARU, C. PETRESCU, G. FLOREA, Switching Solution for Multiple-models Control Systems, Politehnica University Bucharest. 2009b.

6. JANKOVIC, M., M. JANKOVIC, I. KOLMANOVSKY, Constructive Lyapunov Control Design for Turbo Charged Diesel Engines. IEEE Transactions on Control Systems Technology, vol. 8, 2000, pp. 288-299.

7. JUNG, M., Mean-value Modeling and Robust Control of the Air Path of a Turbocharged Diesel Engine, PhD thesis, University of Cambridge, 2003.

8. PAGES, O., P. MOUILlE, B. CARON, Multi-model Control by Applying a Symbolic Fuzzy Switches, IFAC, 2000.

9. POPESCU, D., D. STEFANOIU, Industrial Control, Ed. Agir, Bucharest, Romania, 2007.
10. SODERSTROM, T., P. STOICA, System Identification, Prentice Hall, London, 1989.

11. NARENDRA, K. S., J. BALAKRISHNAN, Adaptive Control using multiple models, IEEE Transactions on Automatic Control, vol. 42, no. 2, February, 1997, pp. 171-187.

12. LUPU C., D. POPESCU, C. PETRESCU, Multi Model Adaptive Control System, ISAS ${ }^{\text {ce }} 99$ Conference, U.S.A., Orlando, 1315 August 1999.

13. LUPU C., D. POPESCU, C. PETRESCU, A1. TICLEA, C. DIMON, A. UDREA, I. BOGDAN, Multiple-Model Design and Switching Solution for Nonlinear Processes Control -, ISC'08, The 6th Annual Industrial Simulation Conference , 09-11 June, 2008, Lyon, France, pp. 71-76, ISBN 978-90-77381-4-03.

14. JENSEN, J. P., A. F. KRISTENSEN, S. C. SORENSON, N. HOUBAK, E. HENDRICKS, Mean Value Modeling of a Small Turbocharged Diesel Engine, SAE Paper 910070, International Congress and Exposition, Detroit, 1991.

15. JUNG, M., K. GLOVER. Calibratable Linear Parameter-varying Control of Turbocharged Diesel Engines. IEEE Transactions on Control Systems Technology, vol. 14, 2006, pp. 45-62.

16. LARSEN, M., M. JANKOVIC, P. V. KOKOTOVIC, Indirect Passivation Design for a Diesel Engine Model. In Proceedings of the 2000 IEEE International Conference on Control Applications, USA, 2000, pp. 309-314.

17. DEC, J. E., K. EPPING, S. M. ACEVES, R. L. BECHTOLD, The Potential of HCCI Combustion for High Efficiency and Low Emissions. Society of Automotive Engineers. 2002-01-1923.

18. JOHANSSON, R., D. BLOM, M. KARLSSON, K. EKHOLM, P. TUNESTAL, HCCI Engine Modelling and Control using Conservation Principles. Society of Automotive Engineers, 2008-01-0789. 
